\title{
Immunohistochemical characterization of granulomatosis with polyangiitis exhibiting spontaneous regression
}

\author{
Yasuhiro Masuta, ${ }^{1}$ Yoriaki Komeda, ${ }^{1}$ Ikue Sekai, ${ }^{1}$ Akane Hara, ${ }^{1}$ Masayuki Kurimoto,${ }^{1}$ Keisuke Yoshikawa, ${ }^{1}$ Yasuo Otsuka, ${ }^{1}$ \\ Ryutaro Takada, ${ }^{1}$ Tomoe Yoshikawa, ${ }^{1}$ Ken Kamata, ${ }^{1}$ Kosuke Minaga, ${ }^{1}$ Osamu Maenishi, ${ }^{2}$ Tomohiro Watanabe, ${ }^{1}$ Masatoshi Kudo ${ }^{1}$
}

\begin{abstract}
Background: Granulomatosis with polyangiitis (GPA) is characterized by granulomatous inflammation, vasculitis, and elevated levels of serum proteinase 3 (PR3)-anti-neutrophil cytoplasmic antibody (PR3-ANCA).

Objective: We tried to characterize immune cells accumulated into the lung lesions of a GPA patient exhibiting spontaneous regression.

Methods: Transbronchial lung biopsy (TBLB) samples were subjected to immunohistochemical analyses.

Results: Multiple lung nodules were detected by CT. TBLB showed granulomatous inflammation and small vessel vasculitis. This case was diagnosed as GPA based on pathological findings and elevation of PR-3 ANCA levels. Spontaneous disappearance of multiple lung nodules was observed in CT. $\mathrm{CD}^{+} \mathrm{T}$ cells and $\mathrm{CD} 20^{+} \mathrm{B}$ cells accumulated in the inflammatory lesions surrounding the vessels whereas granulomatous inflammation was mainly comprised of $\mathrm{CD}^{+} \mathrm{T}$ cells and $\mathrm{CD}^{+} 8^{+}$macrophages, but not B cells or myeloperoxidase ${ }^{+}$neutrophils.
\end{abstract}

Conclusion: We characterized immune cell compositions of the lung lesions of a patient with GPA exhibiting spontaneous regression.

Key words: Granulomatosis with Polyangiitis, Spontaneous Regression, Lung, Immunohistochemistry, PR3-ANCA

From:

${ }^{1}$ Department of Gastroenterology and Hepatology, Kindai University

Faculty of Medicine, Osaka-Sayama, Japan

2 Department of Diagnostic Pathology, Kindai University Faculty of Medicine, Osaka-Sayama, Japan

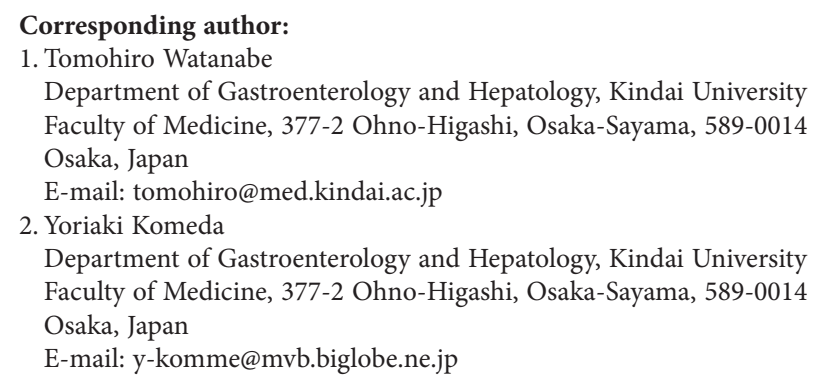

with PR3-ANCA positivity and symptoms related to the respiratory tract and kidney. Patients with GPA are typically treated with immunosuppressants, including glucocorticoids, cyclophosphamide, and rituximab. ${ }^{1,6}$ Without such a treatment, the prognosis of patients with GPA is considered poor. ${ }^{1,6}$ Herein, we report a case of GPA involving the lung and exhibiting spontaneous regression. We performed extensive immunohistochemical (IHC) analyses and found that different types of immune cell populations accumulated in the perivascular and granulomatous lesions in this case. 


\section{Report of Case}

A 24-year-old-man underwent whole-body computed tomography (CT) as his annual blood examinations indicated mild anemia (Hemoglobin, $\mathrm{Hb}, 11 \mathrm{~g} / \mathrm{dL}$ ). Multiple nodules localized in the bilateral peripheral lungs (Figure 1a) were detected in lung CT. He had no history of allergic or autoimmune disorders. Although he experienced several episodes of nasal bleeding, no respiratory, abdominal, and neurologic symptoms were observed. No abnormalities were detected in his nasal mucosa by otorhinolaryngology examination. His stool color was normal. He was admitted to Kindai University Hospital for further examination of multiple lung nodules.

On admission, he was afebrile, and superficial lymph node swelling was not detected. $\mathrm{SpO}_{2}$ was $99 \%$ in the room air. Neither occult blood nor proteinuria was detected in urinalysis. Blood examinations revealed mild anemia and thrombocytosis (Table 1, white blood cell count, $7.45 \times 10^{3} / \mu \mathrm{L}$; red blood cell count, $429 \times 10^{4} / \mu \mathrm{L} ; \mathrm{Hb}, 11.8 \mathrm{~g} / \mathrm{dL}$; hematocrit, $36.5 \%$; platelet count, $47.5 \times 10^{4} / \mu \mathrm{L}$ ). Although blood biochemical analyses indicated that his nutritional state, liver function, and kidney function were normal, the serum concentrations of $\mathrm{Fe}$ and ferritin were low $(\mathrm{Fe}, 28 \mu \mathrm{g} / \mathrm{dL}$, normal range: 70140; ferritin, $17 \mathrm{ng} / \mathrm{mL}, 18-250$ ). The serum concentrations of C-reactive protein and tumor markers, including carcinoembryonic antigen (CEA), carbohydrate antigen 19-9 (CA199), cytokeratin 19-9 fragment (CYFRA), progastrin-releasing peptide (ProGRP), and soluble IL-2 receptor (sIL-2R) were normal. Elevations of total $\operatorname{IgG}(2,149 \mathrm{mg} / \mathrm{dL}$, normal range: 800-1800) and IgE $(634 \mathrm{IU} / \mathrm{mL},<170)$ with normal concentrations of IgA and IgM were observed. Anti-nuclear antibody test results were negative. The serum concentration of myeloperoxidase ANCA (MPO-ANCA) was normal $(1.3 \mathrm{U} / \mathrm{mL}$, normal range: <3.5), whereas that of PR3-ANCA was elevated
(4.5 $\mathrm{U} / \mathrm{mL},<3.5)$. IFN- $\gamma$ release assay for the diagnosis of tuberculosis was negative. Fungal infection was unlikely due to the normal serum concentrations of $\beta$-D-glucan. Positivity of PR3-ANCA and multiple lung nodules led us to consider the possibility of GPA.

18F-fluorodeoxyglucose-positron emission tomography (FDG-PET) revealed a marked uptake of FDG into bilateral lung nodules (SUVmax, 3.5-13.3; Figure 1a). Transbronchial lung biopsy (TBLB) against the right $\mathrm{S} 8$ lung nodule was performed. Granulomatous inflammation, deposition of fibrinoid materials, stenosis of the vascular lumen, and infiltration of immune cells surrounding the small vessels were observed upon hematoxylin and eosin (H\&E) staining (Figure 2a). Deposition of fibrinoid materials and infiltration of immune cells surrounding the small vessels were also detected upon Elastica van Gieson (EVG) staining (Figure 2a). Furthermore, disruption of the vascular wall was clearly visualized by EVG staining (Figure 2a). These pathological findings were consistent with those of GPA. ${ }^{2-4}$ Based on the pathological findings of lung nodules and the positivity of serum PR3-ANCA, the patient was finally diagnosed with GPA. ${ }^{5}$

We then tried to visualize the type of immune cells using IHC analyses, as previously described. ${ }^{7,8} \mathrm{CD}^{+} \mathrm{T}$ cells and $\mathrm{CD} 20^{+} \mathrm{B}$ cells accumulated in the inflammatory lesions surrounding the vessels, whereas little infiltration of $\mathrm{MPO}^{+}$neutrophils was observed (Figure 2b). Granulomatous inflammation mainly comprised $\mathrm{CD}^{+} \mathrm{T}$ cells and $\mathrm{CD} 68^{+}$macrophages, but not $\mathrm{CD} 20^{+} \mathrm{B}$ cells or $\mathrm{MPO}^{+}$neutrophils (Figure $\mathbf{2 b}$ ). Thus, inflammatory lesions surrounding the small vessels were characterized by infiltration of $\mathrm{CD}^{+} \mathrm{T}$ cells and $\mathrm{CD} 20^{+} \mathrm{B}$ cells, whereas granulomatous inflammatory lesions were characterized by infiltration of $\mathrm{CD}^{+} \mathrm{T}$ cells and $\mathrm{CD}^{+} 8^{+}$macrophages. (a)
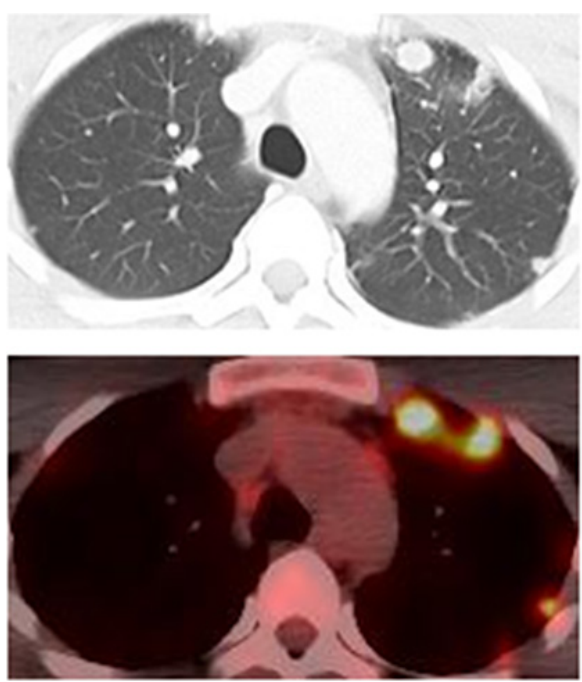
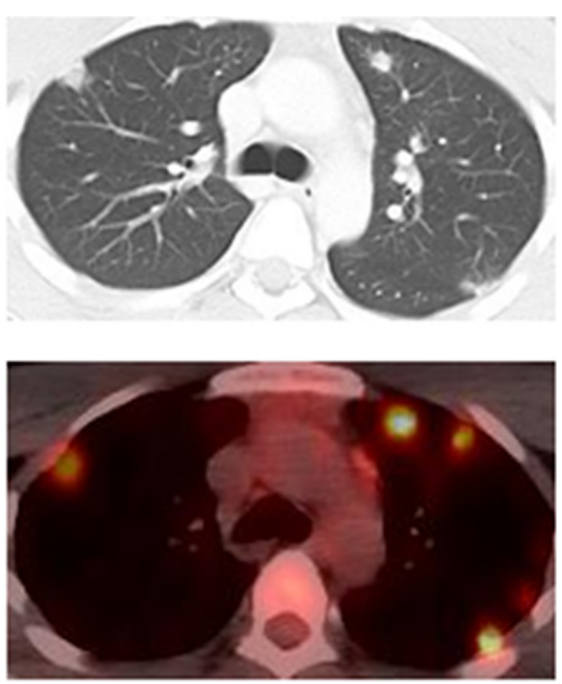

(b)

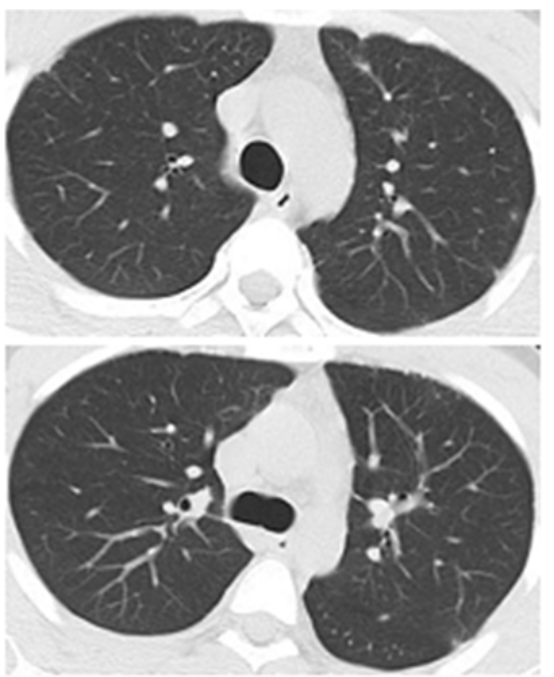

Figure 1. Computed tomography and 18F-fluorodeoxyglucose-positron emission tomography (FDG-PET) exhibiting multiple lung nodules.

(a) Multiple lung nodules were detected in computed tomography. Accumulation of $18 \mathrm{~F}$-fluorodeoxyglucose into these lung nodules was observed in PET.

(b) Computed tomography showed that multiple lung nodules spontaneously disappeared. 
Table 1. Laboratory Data on Admission.

\begin{tabular}{|c|c|c|c|c|c|}
\hline $\mathrm{WBC}\left(\times 10^{3} / \mu \mathrm{L}\right)$ & 7.45 & $\gamma$-GTP $(\mathrm{U} / \mathrm{L})$ & 17 & CEA (ng/mL) & 3.6 \\
\hline $\mathrm{RBC}\left(\times 10^{4} / \mu \mathrm{L}\right)$ & 429 & T-Chol (mg/dL) & 186 & CA19-9 (U/mL) & Not Detected \\
\hline $\mathrm{Hb}(\mathrm{g} / \mathrm{dL})$ & 11.8 & TG (mg/dL) & 71 & ProGRP $(\mathrm{pg} / \mathrm{mL})$ & 34 \\
\hline Ht (\%) & 36.5 & CRP (mg/dL) & 0.578 & CYFRA (ng/mL) & 1.1 \\
\hline $\operatorname{PLT}\left(\times 10^{4} / \mu \mathrm{L}\right)$ & 47.5 & sIL-2R (U/mL) & 593 & $\mathrm{Fe}(\mu \mathrm{g} / \mathrm{dL})$ & 28 \\
\hline $\mathrm{TP}(\mathrm{g} / \mathrm{dL})$ & 8.6 & $\operatorname{IgG}(\mathrm{mg} / \mathrm{gL})$ & 2149 & Ferritin (ng/mL) & 17 \\
\hline Alb (g/dL) & 4.2 & $\operatorname{IgA}(\mathrm{mg} / \mathrm{dL})$ & 275 & $\mathrm{Na}(\mathrm{mmol} / \mathrm{L})$ & 139 \\
\hline T-Bil (mg/dL) & 0.6 & $\operatorname{IgM}(\mathrm{mg} / \mathrm{dL})$ & 94 & $\mathrm{~K}(\mathrm{mmol} / \mathrm{L})$ & 4.1 \\
\hline $\operatorname{ALP}(\mathrm{U} / \mathrm{L})$ & 258 & $\operatorname{IgE}(\mathrm{IU} / \mathrm{mL})$ & 634 & $\mathrm{Cl}(\mathrm{mmol} / \mathrm{L})$ & 103 \\
\hline AST (U/L) & 16 & PR3-ANCA (U/mL) & 4.5 & $\mathrm{Ca}(\mathrm{mg} / \mathrm{dL})$ & 9.7 \\
\hline $\operatorname{ALT}(\mathrm{U} / \mathrm{L})$ & 16 & MPO-ANCA (U/mL) & 1.3 & $\mathrm{BUN}(\mathrm{mg} / \mathrm{dL})$ & 8 \\
\hline $\mathrm{LDH}(\mathrm{U} / \mathrm{L})$ & 184 & $\beta$-D-Glucan (pg/mL) & 8.5 & Cre $(\mathrm{mg} / \mathrm{dL})$ & 0.85 \\
\hline CPK (U/L) & 124 & & & & \\
\hline
\end{tabular}

(a)
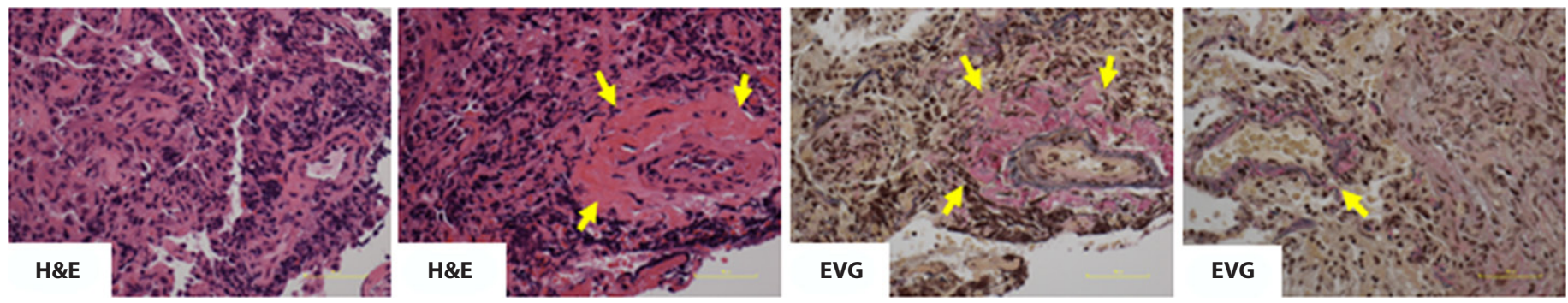

(b)
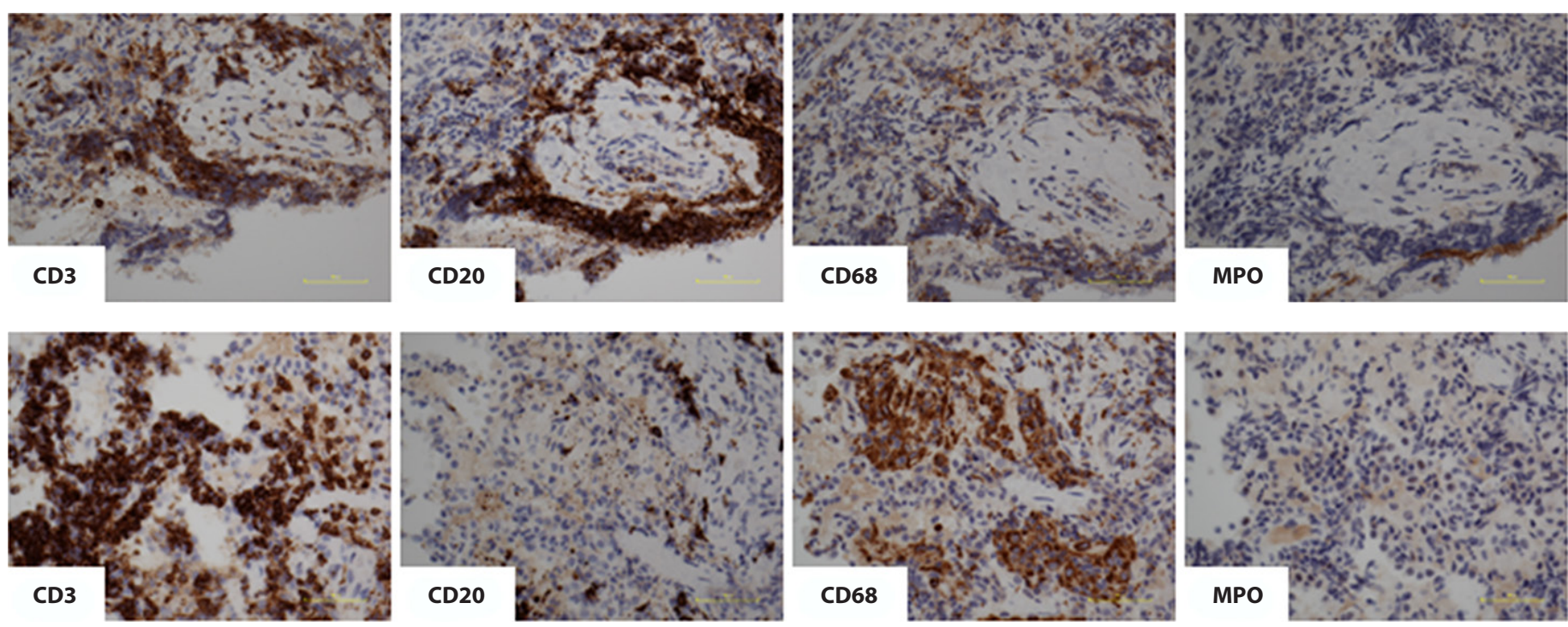

Figure 2. Pathological findings of lung nodules.

(a) Lung biopsy samples were obtained from lung nodules by transbronchial lung biopsy. Lung tissues were subjected to hematoxylin and eosin (H\&E) and Elastica van Gieson (EVG) staining. Granulomatous inflammation ( $1^{\text {st }}$ row), stenosis of the vascular lumen, deposition of fibrinoid materials, and infiltration of immune cells surrounding the vessels $\left(2^{\text {nd }}\right.$ row, arrows) were observed upon H\&E staining. EVG staining revealed deposition of fibrinoid materials, infiltration of immune cells surrounding the vessels ( $3^{\text {rd }}$ row, arrows), and disruption of the vascular wall ( $4^{\text {th }}$ row, arrow). Scale bar: $50 \mu \mathrm{m}$.

(b) Lung biopsy samples were obtained from lung nodules by transbronchial lung biopsy. Lung tissues were subjected to immunohistochemical analyses to visualize $\mathrm{CD}^{+} \mathrm{T}$ cells, $\mathrm{CD} 20^{+} \mathrm{B}$ cells, CD68 ${ }^{+}$macrophages, and myeloperoxidase (MPO)-positive neutrophils. (top panel) Inflammatory lesions surrounding the vessels. (bottom panel) Granulomatous inflammation. Scale bar: $50 \mu \mathrm{m}$. 
Most patients with GPA are typically treated with immunosuppressants, including glucocorticoids, cyclophosphamide, and rituximab. ${ }^{1}$ Although the combination of glucocorticoids with either cyclophosphamide or rituximab is the current gold standard for the treatment of severe AAV, a suitable remedy for asymptomatic AAV remains largely unknown. ${ }^{1}$ Since this patient did not wish to receive immunosuppressive treatment, we decided to follow up carefully without any treatment. Surprisingly, multiple lung nodules completely disappeared four months later in follow-up CT (Figure 1b). Thus, this case of GPA involving the lung exhibited spontaneous regression.

\section{Discussion}

Herein, we report a case of GPA exhibiting spontaneous regression. The following findings from this case met the diagnostic criteria for GPA proposed by Leavitt et al. ${ }^{5}$ First, the patient occasionally presented with bloody nasal discharge. Second, multiple nodules in the peripheral bilateral lungs were detected by CT. Third, pathological examinations using lung biopsy samples revealed granulomatous inflammation and small vessel vasculitis. Fourth, his serum was positive for PR-3 ANCA. Interestingly, spontaneous regression was observed in this case with GPA. Spontaneous regression of lung GPA was also reported by Thomas et al. ${ }^{9}$ Necrotizing granulomatous inflammation scattered multinucleated giant cells and fibrinoid necrosis of small vessels were seen in the first case. To the best of our knowledge, this is the second case of GPA exhibiting spontaneous regression. However, detailed IHC analyses were not performed in the first case exhibiting spontaneous regression of lung GPA.

Predominant infiltration of $\mathrm{CD}^{+} \mathrm{T}$ cells, CD $20^{+} \mathrm{B}$ cells, and $\mathrm{CD} 68^{+}$macrophages in lung inflammatory lesions was observed in our IHC analyses. Although neutrophil activation is considered to play a critical role in the immunopathogenesis of AAV, accumulation of $\mathrm{MPO}^{+}$neutrophils was barely observed in our IHC analyses. ${ }^{1}$ A lack of neutrophil infiltration can be partially explained by the phases of inflammation. The acute lesions of GPA exhibit intense infiltration of activated neutrophils..$^{2-4}$ As the lesions progress, such neutrophil-mediated acute lesions are replaced by chronic lesions characterized by the accumulation of $\mathrm{T}$ cells and macrophages. ${ }^{2-4}$ Based on the predominant infiltration of $\mathrm{T}$ cells and macrophages, our case was considered a chronic phase. Another interesting finding from IHC analyses was a marked difference in the immune cell compositions of the granulomatous and peri-vascular lesions. $\mathrm{CD}^{+} \mathrm{T}$ cells and $\mathrm{CD} 20^{+} \mathrm{B}$ cells accumulated in the peri-vascular lesions, whereas $\mathrm{CD}^{+} \mathrm{T}$ cells and $\mathrm{CD}^{+} 8^{+}$ macrophages constituted the immune cells accumulated in the granulomatous lesions. Therefore, T-B and T-macrophage interactions were likely to be induced in the peri-vascular and granulomatous lesions, respectively. It would be interesting to determine whether such a difference in immune cell networks in granulomatous and peri-vascular lesions is generally exhibited by patients with GPA with spontaneous regression.

A question arising from this case is the mechanisms accounting for spontaneous regression of GPA. Several types of potential risk factors for the development of GPA have been identified. ${ }^{1,6}$ Exposure to environmental and infectious agents and drugs can trigger GPA. ${ }^{1,6}$ Therefore, reduced exposure to these agents might have induced regression of GPA in this case. However, the patient had not been exposed to silica, a prototypical environmental agent for the development of GPA. ${ }^{10}$ As for the possibility of drug-induced GPA, the patient received no medication. ${ }^{11}$ Hence, drug or environmental agent-induced GPA was less likely in this case. However, we could not completely exclude the possibility of infectious agent-triggered GPA. Some studies have focused on the association between nasal carriage of Staphylococcus aureus and GPA flare; however, we did not confirm nasal carriage of this bacterium in this case. ${ }^{11}$ Therefore, we could not identify risk factors associated with the development and regression of GPA.

In conclusion, we report a case of GPA exhibiting spontaneous regression. Different types of immune cell populations were accumulated in the perivascular and granulomatous lesions in this case. Accumulation of $\mathrm{CD}^{+} \mathrm{T}$ cells and $\mathrm{CD} 20^{+}$ $\mathrm{B}$ cells was seen in the inflammatory lesions surrounding the vessels whereas granulomatous inflammation was characterized by accumulation of $\mathrm{CD}^{+} \mathrm{T}$ cells and $\mathrm{CD}^{+} 8^{+}$macrophages. Although GPA generally requires immunosuppression, spontaneous regression of GPA can occur, as in this case. Careful follow-up without administration of immunosuppressants may be a treatment option for asymptomatic and mild GPA.

\section{Acknowledgements}

This work was supported by a grant from Yakult Bio-Science Foundation. Yasuhiro Masuta, Yoriaki Komeda, Ikue Sekai, Akane Hara, Masayuki Kurimoto, Keisuke Yoshikawa, Yasuo Otsuka, Ryutaro Takada, Tomoe Yoshikawa, Ken Kamata, Kosuke Minaga, Osamu Maenishi, Tomohiro Watanabe and Masatoshi Kudo declare that they have no conflicts of interest. Yasuhiro Masuta and Tomohiro Watanabe performed experiments and wrote the manuscript draft. Yasuhiro Masuta, Yoriaki Komeda, Ikue Sekai, Akane Hara, Masayuki Kurimoto, Keisuke Yoshikawa, Yasuo Otsuka and Ryutaro Takada took care of the patient. Tomoe Yoshikawa, Ken Kamata, Kosuke Minaga, and Masatoshi Kudo revised the manuscript. Osamu Maenishi performed pathological diagnosis. We would like to thank Ms. Yukiko Ueno for her secretarial support. We would like to thank Dr. Yusaku Nishikawa for his advice on the diagnosis of GPA.

\section{References}

1. Kitching AR, Anders HJ, Basu N, Brouwer E, Gordon J, Jayne DR, et al. ANCA-associated vasculitis. Nat Rev Dis Primers. 2020;6(1):71.

2. Jennette JC, Falk RJ, Hu P, Xiao H. Pathogenesis of antineutrophil cytoplasmic autoantibody-associated small-vessel vasculitis. Annu Rev Pathol. 2013;8:139-60.

3. Jennette JC, Falk RJ, Bacon PA, Basu N, Cid MC, Ferrario F, et al. 2012 revised International Chapel Hill Consensus Conference Nomenclature of Vasculitides. Arthritis Rheum. 2013;65(1):1-11

4. Jennette JC. Nomenclature and classification of vasculitis: lessons learned from granulomatosis with polyangiitis (Wegener's granulomatosis). Clin Exp Immunol. 2011;164 Suppl 1:7-10.

5. Leavitt RY, Fauci AS, Bloch DA, Michel BA, Hunder GG, Arend WP, et al The American College of Rheumatology 1990 criteria for the classification of Wegener's granulomatosis. Arthritis Rheum. 1990;33(8):1101-7. 
6. Wallace ZS, Stone JH. Personalized Medicine in ANCA-Associated Vasculitis ANCA Specificity as the Guide? Front Immunol. 2019;10:2855.

7. Watanabe T, Sadakane Y, Yagama N, Sakurai T, Ezoe H, Kudo M, et al. Nucleotide-binding oligomerization domain 1 acts in concert with the cholecystokinin receptor agonist, cerulein, to induce IL-33-dependent chronic pancreatitis. Mucosal Immunol. 2016;9(5):1234-49.

8. Watanabe T, Yamashita K, Arai Y, Minaga K, Kamata K, Nagai T, et al. Chronic Fibro-Inflammatory Responses in Autoimmune Pancreatitis Depend on IFN-alpha and IL-33 Produced by Plasmacytoid Dendritic Cells. J Immunol. 2017;198(10):3886-96.
9. Thomas J, Gaffney JK. Spontaneous remission in Wegener's granulomatosis. Scand J Rheumatol. 2007;36(4):324-6.

10. Gomez-Puerta JA, Gedmintas L, Costenbader KH. The association between silica exposure and development of ANCA-associated vasculitis: systematic review and meta-analysis. Autoimmun Rev. 2013;12(12):1129-35.

11. Choi HK, Merkel PA, Walker AM, Niles JL. Drug-associated antineutrophil cytoplasmic antibody-positive vasculitis: prevalence among patients with high titers of antimyeloperoxidase antibodies. Arthritis Rheum. 2000;43(2):405-13. 